\title{
Moudafi's Viscosity Approximations with Demi-Continuous and Strong Pseudo-Contractions for Non-Expansive Semigroups
}

\author{
Changqun Wu, ${ }^{1}$ Sun Young Cho, ${ }^{2}$ and Meijuan Shang ${ }^{3}$ \\ ${ }^{1}$ School of Business and Administration, Henan University, Kaifeng 475001, China \\ 2 Department of Mathematics, Gyeongsang National University, Jinju 660-701, South Korea \\ ${ }^{3}$ Department of Mathematics, Shijiazhuang University, Shijiazhuang 050035, China
}

Correspondence should be addressed to Changqun Wu, kyls2003@yahoo.com.cn

Received 6 October 2009; Accepted 10 December 2009

Academic Editor: Yeol J. E. Cho

Copyright (c) 2010 Changqun Wu et al. This is an open access article distributed under the Creative Commons Attribution License, which permits unrestricted use, distribution, and reproduction in any medium, provided the original work is properly cited.

We consider viscosity approximation methods with demi-continuous strong pseudo-contractions for a non-expansive semigroup. Strong convergence theorems of the purposed iterative process are established in the framework of Hilbert spaces.

\section{Introduction and Preliminaries}

Throughout this paper, we assume that $H$ is a real Hilbert space and denote by $\mathbb{R}^{+}$the set of nonnegative real numbers. Let $C$ be a nonempty closed and convex subset of $H$ and $T: C \rightarrow$ $C$ a nonlinear mapping. We use $F(T)$ to denote the fixed point set of $T$.

Recall that the mapping $T$ is said to be an $\alpha$-contraction if there exists a constant $\alpha \in$ $(0,1)$ such that

$$
\|f(x)-f(y)\| \leq \alpha\|x-y\|, \quad \forall x, y \in C .
$$

$T$ is said to be non-expansive if

$$
\|T x-T y\| \leq\|x-y\|, \quad \forall x, y \in C .
$$


$T$ is said to be strictly pseudo-contractive if there exists a constant $\alpha \in[0,1)$ such that

$$
\|T x-T y\|^{2} \leq\|x-y\|^{2}+\alpha\|(I-T) x-(I-T) y\|^{2}, \quad \forall x, y \in C
$$

Note that the class of strict pseudo-contractions strictly includes the class of non-expansive mappings as a special case. That is, $T$ is non-expansive if and only if the coefficient $\alpha=0$. It is also said to be pseudo-contractive if $\alpha=1$. That is,

$$
\|T x-T y\|^{2} \leq\|x-y\|^{2}+\|(I-T) x-(I-T) y\|^{2}, \quad \forall x, y \in C .
$$

$T$ is said to be strongly pseudo-contractive if there exists a positive constant $\lambda \in(0,1)$ such that $T+\lambda I$ is pseudo-contractive. Clearly, the class of strict pseudo-contractions falls into the one between classes of non-expansive mappings and pseudo-contractions. We remark also that the class of strongly pseudo-contractive mappings is independent of the class of strict pseudo-contractions (see, e.g., $[1,2]$ ).

The following examples are due to Chidume and Mutangadura [3] and Zhou [4].

Example 1.1. Take $C=(0, \infty)$ and define $T: C \rightarrow C$ by

$$
T x=\frac{x^{2}}{1+x}
$$

Then $T$ is a strict pseudo-contraction but not a strong pseudo-contraction.

Example 1.2. Take $C=\mathbb{R}^{1}$ and define $T: C \rightarrow C$ by

$$
T x= \begin{cases}1, & x \in(-\infty,-1) \\ \sqrt{1-(1+x)^{2},} & x \in[-1,0) \\ -\sqrt{1-(x-1)^{2}}, & x \in[0,1] \\ -1, & x \in(1, \infty) .\end{cases}
$$

Then, $T$ is a strong pseudo-contraction but not a strict pseudo-contraction.

Example 1.3. Take $H=\mathbb{R}^{2}$ and $B=\left\{x \in \mathbb{R}^{2}:\|x\| \leq 1\right\}, B_{1}=\{x \in B:\|x\| \leq 1 / 2\}, B_{2}=\{x \in B$ : $1 / 2 \leq\|x\| \leq 1\}$. If $x \in(a, b) \in H$, we define $x^{\perp}$ to be $(b,-a) \in H$. Define $T: B \rightarrow B$ by

$$
T x= \begin{cases}x+x^{\perp}, & x \in B_{1}, \\ \frac{x}{\|x\|}-x+x^{\perp}, & x \in B_{2} .\end{cases}
$$

Then, $T$ is a Lipschitz pseudo-contraction but not a strict pseudo-contraction.

It is very clear that, in a real Hilbert space $H,(1.4)$ is equivalent to

$$
\langle T x-T y, x-y\rangle \leq\|x-y\|^{2} .
$$


$T$ is strongly pseudo-contractive if and only if there exists a positive constant $\lambda \in(0,1)$ such that

$$
\langle T x-T y, x-y\rangle \leq(1-\lambda)\|x-y\|^{2} .
$$

for all $x, y \in C$.

Let $\mathcal{F}=\{T(t): t \geq 0\}$ be a strongly continuous semigroups of non-expansive mappings on a closed convex subset $C$ of a Hilbert space $H$, that is,

(a) for each $t \in \mathbb{R}^{+}, T(t)$ is a non-expansive mapping on $C$;

(b) $T(0) x=x$ for all $x \in C$;

(c) $T(s+t)=T(s) T(t)$ for all $s, t \geq 0$;

(d) for each $x \in H$, the mapping $T(\cdot) s$ from $\mathbb{R}^{+}$into $C$ is continuous.

We denote by $F$ the set of common fixed points of $F$, that is,

$$
F:=\{x \in C: T(t) x=x, t>0\}=\bigcap_{t>0} F(\mathrm{~T}(t))
$$

We know that $F$ is nonempty if $C$ is bounded (see [5]). In [6], Shioji and Takahashi proved the following theorem:

Theorem ST. Let $C$ be a closed convex subset of a Hilbert space $H$. Let $\{T(t): t \geq 0\}$ be a strongly continuous semigroup of non-expansive mappings on $C$ such that $\bigcap_{t \geq 0} F(T(t)) \neq \emptyset$. Let $\left\{\alpha_{n}\right\}$ and $\left\{t_{n}\right\}$ be sequences of real numbers satisfying $0<\alpha_{n}<1, \lim _{n \rightarrow \infty} \alpha_{n}=0, t_{n} \geq 0$ and $\lim _{n \rightarrow \infty} t_{n}=\infty$. Fix $u \in C$ and define a sequence $\left\{x_{n}\right\}$ in $C$ by

$$
x_{n}=\alpha_{n} u+\left(1-\alpha_{n}\right) \frac{1}{t_{n}} \int_{0}^{t_{n}} T(s) x_{n} d s, \quad \forall n \geq 1 .
$$

Then $\left\{x_{n}\right\}$ converges strongly to the element of $F$ nearest to $u$. theorem:

Suzuki [9] improved the results of Shioji and Takahashi [6] and proved the following

Theorem S. Let $C$ be a closed convex subset of a Hilbert space $H$. Let $\{T(t): t \geq 0\}$ be a strongly continuous semigroup of non-expansive mappings on $C$ such that $\bigcap_{t \geq 0} F(T(t)) \neq \emptyset$. Let $\alpha_{n}$ and $\left\{t_{n}\right\}$ be sequences of real numbers satisfying $0<\alpha_{n}<1, t_{n}>0$ and $\lim _{n \rightarrow \infty} t_{n}=\lim _{n \rightarrow \infty} \alpha_{n} / t_{n}=0$. Fix $u \in C$ and define a sequence $\left\{x_{n}\right\}$ in $C$ by

$$
x_{n}=\alpha_{n} u+\left(1-\alpha_{n}\right) T\left(t_{n}\right) x_{n}, \quad \forall n \geq 1
$$

Then $\left\{x_{n}\right\}$ converges strongly to the element of $F$ nearest to $u$.

Recently, The so-called viscosity approximation methods have been studied by many author. They are very important because they are applied to convex optimization, linear programming, monotone inclusions and elliptic differential equations. In [8], Moudafi 
proposed the viscosity approximation method of selecting a particular fixed point of a given non-expansive mapping in Hilbert spaces. If $H$ is a Hilbert space, $T: C \rightarrow C$ is a nonexpansive self-mapping on a nonempty closed convex $C$ of $H$ and $f: C \rightarrow C$ is a contraction, he proved the following result.

Theorem M. The sequence $\left\{x_{n}\right\}$ generated by the scheme

$$
x_{n}=\frac{1}{1+\epsilon_{n}} T x_{n}+\frac{\epsilon_{n}}{1+\epsilon_{n}} f\left(x_{n}\right)
$$

converges strongly to the unique solution of the variational inequality:

$$
\bar{x} \in F(T), \text { such that }\langle(I-f) \bar{x}, \bar{x}-x\rangle \leq 0, \quad \forall x \in F(T),
$$

where $\left\{\epsilon_{n}\right\}$ is a sequence of positive numbers tending to zero.

In this paper, motivated by Moudafi [8], Shioji and Takahashi [6], Suzuki [9] and Xu [10], we introduce the following implicit iterative scheme:

$$
x_{n}=\alpha_{n} f\left(x_{n}\right)+\left(1-\alpha_{n}\right) T\left(t_{n}\right) x_{n}, \quad \forall n \geq 1,
$$

where $f$ is a demi-continuous and strong pseudo-contraction, and prove that the sequence $\left\{x_{n}\right\}$ generated by the above iterative process converge strongly to a common fixed point $q \in F$. Also, we show that the point $q$ solves the variational inequality

$$
\langle(f-I) q, x-q\rangle \leq 0, \quad \forall x \in F .
$$

Our results mainly improve and extend the corresponding results announced by Moudafi [8], Shioji and Takahashi [6], Suzuki [9], Xu [10] and some others.

In order to prove our main result, we need the following lemmas and definitions:

Let $X, Y$ be linear normed spaces. $T: D(T) \subset X \rightarrow Y$ is said to be demi-continuous if, for any $\left\{x_{n}\right\} \subset D(T)$ we have $T x_{n} \rightarrow T x_{0}$ as $x_{n} \rightarrow x_{0}$, where $\rightarrow$ and $\rightarrow$ denote strong and weak convergence, respectively.

Recall that a space $X$ satisfies Opial's condition [11] if, for each sequence $\left\{x_{n}\right\}$ in $X$ which converges weakly to point $x \in X$,

$$
\liminf _{n \rightarrow \infty}\left\|x_{n}-x\right\|<\liminf _{n \rightarrow \infty}\left\|x_{n}-y\right\|, \quad \forall y \in X(y \neq x)
$$

Lemma 1.4. Let $C$ be a closed convex subset of a Hilbert space $H, f: C \rightarrow C$ be a strong pseudocontraction with the coefficient $0<\alpha<1$. Then

$$
\langle x-y,(I-f) x-(I-f) y\rangle \geq(1-\alpha)\|x-y\|^{2}, \quad \forall x, y \in C .
$$

That is, $I-f$ is strongly monotone with the coefficient $1-\alpha$. 
Proof. From the definition of strongly pseudo-contractions, one sees that

$$
\langle x-y, f x-f y\rangle \leq \alpha\|x-y\|^{2} .
$$

Therefore, we have

$$
\begin{aligned}
\left\langle x-y_{,}(I-f) x-(I-f) y\right\rangle & =\langle x-y, x-y\rangle-\langle x-y, f x-f y\rangle \\
& \geq(1-\alpha)\|x-y\|^{2}, \quad \forall x, y \in C .
\end{aligned}
$$

This completes the proof.

Remark 1.5. If $f$ is non-expansive, then

$$
\langle x-y,(I-f) x-(I-f) y\rangle \geq 0, \quad \forall x, y \in C .
$$

\section{Main Results}

First, we give a convergence theorem for a non-expansive semigroup by Moudafi's viscosity approximation methods with $\alpha$-contractions.

Theorem 2.1. Let $C$ be a nonempty closed and convex subset of a Hilbert space $H$. Let $\{T(t): t \geq 0\}$ be a strongly continuous semigroup of non-expansive mappings from $C$ into itself such that $F \neq \emptyset$. Let $f: C \rightarrow C$ be an $\alpha$-contraction. Let $\left\{\alpha_{n}\right\}$ and $\left\{t_{n}\right\}$ be sequences of real numbers satisfying $0<\alpha_{n}<1$, $t_{n}>0$ and $\lim _{n \rightarrow \infty} t_{n}=\lim _{n \rightarrow \infty} \alpha_{n} / t_{n}=0$. Define a sequence $\left\{x_{n}\right\}$ in the following manner:

$$
x_{1} \in C, \quad x_{n}=\alpha_{n} f\left(x_{n}\right)+\left(1-\alpha_{n}\right) T\left(t_{n}\right) x_{n}, \quad \forall n \geq 1 .
$$

Then $\left\{x_{n}\right\}$ converges strongly to $p \in F$ which solves the following variational inequality:

$$
\langle(I-f) p, p-x\rangle \leq 0, \quad \forall x \in F .
$$

Proof. Define a sequence $\left\{y_{n}\right\}$ in the following manner

$$
y_{1} \in C, \quad y_{n}=\alpha_{n} f(p)+\left(1-\alpha_{n}\right) T\left(t_{n}\right) y_{n}, \quad \forall n \geq 1
$$

From Theorem S, one sees that

$$
\operatorname{Lim}_{n \rightarrow \infty} y_{n}=P_{C} f(p)=p
$$

Therefore, it is sufficient to prove that

$$
\lim _{n \rightarrow \infty}\left\|y_{n}-x_{n}\right\|=0
$$


Noticing that

$$
\begin{aligned}
\left\|x_{n}-y_{n}\right\| & =\left\|\alpha_{n}\left[f\left(x_{n}\right)-f(p)\right]+\left(1-\alpha_{n}\right)\left[T\left(t_{n}\right) x_{n}-T\left(t_{n}\right) y_{n}\right]\right\| \\
& \leq \alpha_{n} \alpha\left\|x_{n}-p\right\|+\left(1-\alpha_{n}\right)\left\|x_{n}-y_{n}\right\|,
\end{aligned}
$$

one has

$$
\left\|x_{n}-y_{n}\right\| \leq \alpha\left\|x_{n}-p\right\| \leq \alpha\left\|x_{n}-y_{n}\right\|+\alpha\left\|y_{n}-p\right\|
$$

It follows that

$$
\left\|x_{n}-y_{n}\right\| \leq \frac{\alpha}{1-\alpha}\left\|y_{n}-p\right\|
$$

From (2.4), one obtains that (2.5) holds. This completes the proof.

Remark 2.2. If $f(x)=u \in C$, a fixed point, for all $x \in C$, then Theorem 2.1 is reduced to Suzuki's results [9]. Theorem 2.1 also can be viewed as an improvement of the corresponding results in Shioji and Takahashi [6].

The class of pseudo-contractions is one of the most important classes of mappings among nonlinear mappings. Browder [1] proved the first existence result of fixed point for demi-continuous pseudo-contractions in the framework of Hilbert space. During the past 40 years or so, mathematicians have been devoting to the studies on the existence and convergence of fixed points of nonexpansive mappings and pseudo-contractive mappings. See, for example, [1-26].

Assume that $P$ is a metric projection from a Hilbert space to its nonempty closed convex subset $C$ and $f: C \rightarrow C$ is a $\alpha$-contraction. It is easy to see that the mapping $P f$ has a unique fixed point in $C$. That is why Theorem 2.1 can be deduced from Theorem $S$ easily. What happens if we relax $f$ from $\alpha$-contraction to strong pseudo-contraction? Does Theorem 2.1 still holds if $f$ is a strong pseudo-contraction? Since we don't know whether the mapping $P f$, where $f$ is a strong pseudo-contraction, has a unique fixed point or not, we can not get the desired results from Theorem S.

Next, we give the second convergence theorem for the non-expansive semigroup by Moudafi's viscosity approximation methods with strong pseudo-contractions.

Theorem 2.3. Let $C$ be a nonempty closed and convex subset of a Hilbert space $H$. Let $\{T(t): t \geq 0\}$ be a strongly continuous semigroup of non-expansive mappings from $C$ into itself such that $F \neq \emptyset$. Let $f: C \rightarrow C$ be bounded, demi-continuous and strong pseudo-contraction with the coefficient $\alpha \in(0,1)$. Let $\left\{\alpha_{n}\right\}$ and $\left\{t_{n}\right\}$ be sequences of real numbers satisfying $0<\alpha_{n}<1, t_{n}>0$ and $\lim _{n \rightarrow \infty} t_{n}=\lim _{n \rightarrow \infty} \alpha_{n} / t_{n}=0$. Define a sequence $\left\{z_{n}\right\}$ by the following manner:

$$
z_{1} \in C, \quad z_{n}=\alpha_{n} f\left(z_{n}\right)+\left(1-\alpha_{n}\right) T\left(t_{n}\right) z_{n}, \quad \forall n \geq 1
$$

Then $\left\{z_{n}\right\}$ converges strongly to $q \in F$ which solves the following variational inequality:

$$
\langle(I-f) q, q-z\rangle \leq 0, \quad \forall z \in F .
$$


Proof. First, we show that the fixed point equation (2.10) is well-defined. For any $n \geq 1$, define a mapping $T_{n}$ as follows

$$
T_{n} z=\alpha_{n} f(z)+\left(1-\alpha_{n}\right) T\left(t_{n}\right) z
$$

For any $x, y \in C$, one has

$$
\begin{aligned}
\left\langle T_{n} x-T_{n} y, x-y\right\rangle & =\alpha_{n}\langle f(x)-f(y), x-y\rangle+\left(1-\alpha_{n}\right)\left\langle T\left(t_{n}\right) x-T\left(t_{n}\right) y, x-y\right\rangle \\
& \leq \alpha_{n} \alpha\|x-y\|^{2}+\left(1-\alpha_{n}\right)\|x-y\|^{2}=\left[1-\alpha_{n}(1-\alpha)\right]\|x-y\|^{2}
\end{aligned}
$$

This shows $T_{n}$ is demi-continuous and strong pseudo-contraction with the efficient $1-\alpha_{n}(1-$ $\alpha$ ). From Lan and $\mathrm{Wu}\left[17\right.$, Theorem 2.2], one sees that $T_{n}$ has a unique fixed point, denoted $z_{n}$, which uniquely solves the fixed point equation

$$
z_{n}=\alpha_{n} f\left(z_{n}\right)+\left(1-\alpha_{n}\right) T\left(t_{n}\right) z_{n}
$$

This is, (2.9) is well-defined. The uniqueness of the solution of the variational inequality (2.10) is a consequence of the strong monotonicity of $I-f$. Suppose $p, q \in F$ both are solutions to (2.10). It follows that

$$
\begin{aligned}
& \langle(I-f) p, p-q\rangle \leq 0 \\
& \langle(I-f) q, q-p\rangle \leq 0 .
\end{aligned}
$$

Adding up (2.14), one obtains

$$
\langle(I-f) p-(I-f) q, p-q\rangle \leq 0, \quad \forall x \in F
$$

Since Lemma 1.4, one sees that $p=q$. Next, we use $q$ to denote the unique solution of the variational inequality (2.10).

Next, we show that $\left\{z_{n}\right\}$ is bounded. Indeed, for any $z \in F$, we have

$$
\begin{aligned}
\left\|z_{n}-z\right\|^{2}= & \left\langle\alpha_{n}\left(f\left(z_{n}\right)-z\right)+\left(1-\alpha_{n}\right)\left(T\left(t_{n}\right) z_{n}-z\right), z_{n}-z\right\rangle \\
= & \alpha_{n}\left\langle f\left(z_{n}\right)-f(z), z_{n}-z\right\rangle+\alpha_{n}\left\langle f(z)-z, z_{n}-z\right\rangle \\
& +\left\langle\left(1-\alpha_{n}\right)\left(T\left(t_{n}\right) z_{n}-z, z_{n}-z\right\rangle\right. \\
\leq & \alpha_{n} \alpha\left\|z_{n}-z\right\|^{2}+\alpha_{n}\left\langle f(z)-z, z_{n}-z\right\rangle+\left(1-\alpha_{n}\right)\left\|z_{n}-z\right\|^{2}
\end{aligned}
$$

from which it follows that

$$
\left\|z_{n}-z\right\|^{2} \leq \frac{1}{1-\alpha}\left\langle f(z)-z, z_{n}-z\right\rangle .
$$


That is,

$$
\left\|z_{n}-z\right\| \leq \frac{1}{1-\alpha}\|f(z)-z\|
$$

This implies that $\left\{z_{n}\right\}$ is bounded. Let $\left\{z_{n_{i}}\right\}$ be an arbitrary subsequence of $\left\{z_{n}\right\}$. Then there exists a subsequence $\left\{z_{n_{i_{j}}}\right\}$ of $\left\{z_{n_{i}}\right\}$ which converges weakly to a point $q$.

Next, we show that $q \in F$. In fact, put $x_{j}=z_{n_{i_{j}}}, \gamma_{j}=\alpha_{n_{i_{j}}}$ and $s_{j}=t_{n_{i_{j}}}$ for all $j \geq 1$. Fix $t>0$. Noticing that

$$
\begin{aligned}
\left\|x_{j}-T(t) q\right\| \leq & \sum_{k=0}^{\left[t / s_{j}\right]-1}\left\|T\left((k+1) s_{j}\right) x_{j}-T\left(k s_{j}\right) x_{j}\right\| \\
& +\left\|T\left(\left[\frac{t}{s_{j}}\right] s_{j}\right) x_{j}-T\left(\left[\frac{t}{s_{j}}\right] s_{j}\right) q\right\|+\left\|T\left(\left[\frac{t}{s_{j}}\right] s_{j}\right) q-T(t) q\right\| \\
\leq & {\left[\frac{t}{s_{j}}\right]\left\|T\left(s_{j}\right) x_{j}-x_{j}\right\|+\left\|x_{j}-q\right\|+\left\|T\left(t-\left[\frac{t}{s_{j}}\right] s_{j}\right) q-q\right\| } \\
\leq & \frac{r_{j}}{s_{j}} t\left\|A T\left(s_{j}\right) x_{j}-\gamma f\left(x_{j}\right)\right\|+\left\|x_{j}-q\right\| \\
& +\max \left\{\|T(s) q-q\|: 0 \leq s \leq s_{j}\right\}, \quad \forall j \geq 1,
\end{aligned}
$$

we have

$$
\liminf _{j \rightarrow \infty}\left\|x_{j}-T(t) q\right\| \leq \liminf _{j \rightarrow \infty}\left\|x_{j}-q\right\|
$$

From Opial's condition, we have $T(t) q=q$. Therefore, $q \in F$. In the inequality (2.17), replacing $p$ with $x$, we have

$$
\left\|x_{j}-q\right\|^{2} \leq \frac{1}{1-\alpha}\left\langle f(q)-q, x_{j}-q\right\rangle
$$

Taking the limit as $j \rightarrow \infty$ in (2.21), we obtain

$$
\lim _{j \rightarrow \infty}\left\|x_{j}-q\right\|=0
$$

Since the subsequence $\left\{z_{n_{i}}\right\}$ is arbitrary, it follows that $\left\{z_{n}\right\}$ converges strongly to $q$.

Finally, we prove that $q \in F$ is a solution of the variational inequality (2.10). From (2.9), one sees

$$
(I-f) z_{n}=-\frac{1}{\alpha_{n}}\left(1-\alpha_{n}\right)\left[z_{n}-T\left(t_{n}\right) z_{n}\right]
$$


For any $z \in F$, it follows from (1.21) that

$$
\begin{aligned}
\left\langle(I-f) z_{n}, z_{n}-z\right\rangle= & -\frac{1}{\alpha_{n}}\left\langle\left(1-\alpha_{n}\right)\left[z_{n}-T\left(t_{n}\right) z_{n}\right], z_{n}-z\right\rangle \\
= & -\frac{1}{\alpha_{n}}\left\langle\left[I-T\left(t_{n}\right)\right] z_{n}-\left[I-T\left(t_{n}\right)\right] z, z_{n}-z\right\rangle \\
& +\left\langle\left[I-T\left(t_{n}\right)\right] z_{n}, z_{n}-z\right\rangle \\
\leq & \left\langle\left[I-T\left(t_{n}\right)\right] z_{n}, z_{n}-z\right\rangle \\
= & \left\langle\alpha_{n} f\left(z_{n}\right)-\alpha_{n} T\left(t_{n}\right) z_{n}, z_{n}-z\right\rangle \\
= & \alpha_{n}\left\langle f\left(z_{n}\right)-T\left(t_{n}\right) z_{n}, z_{n}-z\right\rangle .
\end{aligned}
$$

Letting $n \rightarrow \infty$, one sees

$$
\langle(I-f) q, q-z\rangle \leq 0, \quad \forall z \in F
$$

That is, $q \in F$ is the unique solution to the variational inequality (2.10). This completes the proof.

Remark 2.4. From Theorem 2.3, we see that the composite mapping $P_{C} f$ has a unique fixed point in $C$.

\section{References}

[1] F. E. Browder, "Fixed-point theorems for noncompact mappings in Hilbert space," Proceedings of the National Academy of Sciences of the United States of America, vol. 53, pp. 1272-1276, 1965.

[2] F. E. Browder and W. V. Petryshyn, "Construction of fixed points of nonlinear mappings in Hilbert space," Journal of Mathematical Analysis and Applications, vol. 20, pp. 197-228, 1967.

[3] C. E. Chidume and S. A. Mutangadura, "An example of the Mann iteration method for Lipschitz pseudocontractions," Proceedings of the American Mathematical Society, vol. 129, no. 8, pp. 2359-2363, 2001.

[4] H. Zhou, "Convergence theorems of fixed points for Lipschitz pseudo-contractions in Hilbert spaces," Journal of Mathematical Analysis and Applications, vol. 343, no. 1, pp. 546-556, 2008.

[5] F. E. Browder, "Nonexpansive nonlinear operators in a Banach space," Proceedings of the National Academy of Sciences of the United States of America, vol. 54, pp. 1041-1044, 1965.

[6] N. Shioji and W. Takahashi, "Strong convergence theorems for asymptotically nonexpansive semigroups in Hilbert spaces," Nonlinear Analysis: Theory, Methods E Applications, vol. 34, no. 1, pp. 87-99, 1998.

[7] X. Qin, Y. J. Cho, S. M. Kang, and H. Zhou, "Convergence theorems of common fixed points for a family of Lipschitz quasi-pseudocontractions," Nonlinear Analysis: Theory, Methods E Applications, vol. 71, no. 1-2, pp. 685-690, 2009.

[8] A. Moudafi, "Viscosity approximation methods for fixed-points problems," Journal of Mathematical Analysis and Applications, vol. 241, no. 1, pp. 46-55, 2000.

[9] T. Suzuki, "On strong convergence to common fixed points of nonexpansive semigroups in Hilbert spaces," Proceedings of the American Mathematical Society, vol. 131, no. 7, pp. 2133-2136, 2003.

[10] H.-K. Xu, "Viscosity approximation methods for nonexpansive mappings," Journal of Mathematical Analysis and Applications, vol. 298, no. 1, pp. 279-291, 2004.

[11] Z. Opial, "Weak convergence of the sequence of successive approximations for nonexpansive mappings," Bulletin of the American Mathematical Society, vol. 73, pp. 591-597, 1967. 
[12] F. E. Browder, "Convergence of approximants to fixed points of nonexpansive non-linear mappings in Banach spaces," Archive for Rational Mechanics and Analysis, vol. 24, pp. 82-90, 1967.

[13] R. E. Bruck Jr., "A strongly convergent iterative solution of $0 \in U x$ for a maximal monotone operator $U$ in Hilbert space," Journal of Mathematical Analysis and Applications, vol. 48, pp. 114-126, 1974.

[14] C. E. Chidume and H. Zegeye, "Approximate fixed point sequences and convergence theorems for Lipschitz pseudocontractive maps," Proceedings of the American Mathematical Society, vol. 132, no. 3, pp. 831-840, 2004.

[15] Y. J. Cho and X. Qin, "Viscosity approximation methods for a family of $m$-accretive mappings in reflexive Banach spaces," Positivity, vol. 12, no. 3, pp. 483-494, 2008.

[16] K. Deimling, "Zeros of accretive operators," Manuscripta Mathematica, vol. 13, pp. 365-374, 1974.

[17] K. Q. Lan and J. H. Wu, "Convergence of approximants for demicontinuous pseudo-contractive maps in Hilbert spaces," Nonlinear Analysis: Theory, Methods \& Applications, vol. 49, no. 6, pp. 737-746, 2002.

[18] X. Qin and Y. Su, "Approximation of a zero point of accretive operator in Banach spaces," Journal of Mathematical Analysis and Applications, vol. 329, no. 1, pp. 415-424, 2007.

[19] X. Qin and Y. Su, "Strong convergence theorems for relatively nonexpansive mappings in a Banach space," Nonlinear Analysis: Theory, Methods E Applications, vol. 67, no. 6, pp. 1958-1965, 2007.

[20] X. Qin, Y. J. Cho, S. M. Kang, and M. Shang, "A hybrid iterative scheme for asymptotically $k$-strict pseudo-contractions in Hilbert spaces," Nonlinear Analysis: Theory, Methods \& Applications, vol. 70, no. 5, pp. 1902-1911, 2009.

[21] X. Qin, S. M. Kang, and M. Shang, "Strong convergence theorems for accretive operators in Banach spaces," Fixed Point Theory, vol. 9, no. 1, pp. 243-258, 2008.

[22] X. Qin, Y. J. Cho, J. I. Kang, and S. M. Kang, "Strong convergence theorems for an infinite family of nonexpansive mappings in Banach spaces," Journal of Computational and Applied Mathematics, vol. 230, no. 1, pp. 121-127, 2009.

[23] B. E. Rhoades, "Fixed point iterations using infinite matrices," Transactions of the American Mathematical Society, vol. 196, pp. 161-176, 1974.

[24] H. Zhou, "Convergence theorems of common fixed points for a finite family of Lipschitz pseudocontractions in Banach spaces," Nonlinear Analysis: Theory, Methods \& Applications, vol. 68, no. 10, pp. 2977-2983, 2008.

[25] H. Zhou, "Strong convergence of an explicit iterative algorithm for continuous pseudo-contractions in Banach spaces," Nonlinear Analysis: Theory, Methods E Applications, vol. 70, no. 11, pp. 4039-4046, 2009.

[26] H. Zhou, "Demiclosedness principle with applications for asymptotically pseudo-contractions in Hilbert spaces," Nonlinear Analysis: Theory, Methods E Applications, vol. 70, no. 9, pp. 3140-3145, 2009. 